\title{
Philosophiques
}

\section{Les conditions proto-logiques des langues naturelles}

\section{Gilles-Gaston Granger}

Volume 16, numéro 2, automne 1989

URI : https://id.erudit.org/iderudit/027080ar

DOI : https://doi.org/10.7202/027080ar

Aller au sommaire du numéro

Éditeur(s)

Société de philosophie du Québec

ISSN

0316-2923 (imprimé)

1492-1391 (numérique)

Découvrir la revue

Citer cet article

Granger, G.-G. (1989). Les conditions proto-logiques des langues naturelles. Philosophiques, 16(2), 245-256. https://doi.org/10.7202/027080ar

\section{Résumé de l'article}

Peut-on formuler des conditions non empiriques pour qu'un objet ou un fait soit reconnu comme ayant fonction de symbole ? Si oui, il conviendrait de les nommer conditions proto-logiques, car elles concernent des formes, comme la logique, mais sont plus primitives que les déterminations logiques mêmes. L'objet de cet article est de discuter cinq notions qui peuvent être présentées comme candidates à cette fonction d'universels proto- logiques pour les langues naturelles : la pluralité des niveaux d'« articu- lation», l'énoncé complet, la notion d'« ancrage» de l'énoncé à renonciation, le nom propre, la corrélation rhème-thème. De tels universaux ne sont pas présentés comme des classes de symboles, mais des types de fonctions diversement réalisées dans des structures grammaticales. Les mettre en évidence - ou en découvrir d'autres - devrait être l'une des tâches de la philosophie du langage dans sa relation à la science linguistique. 
PHILOSOPHIQUES, Vol. XVI, Numéro 2, Automne 1989

\title{
ARTICLES
}

\section{LES CONDITIONS PROTO-LOGIQUES DES LANGUES NATURELLES ${ }^{\text {1 }}$}

\author{
par Gilles-Gaston Granger
}

RÉSUMÉ. Peut-on formuler des conditions non empiriques pour qu'un objet ou un fait soit reconnu comme ayant fonction de symbole? Si oui, il conviendrait de les nommer conditions proto-logiques, car elles concernent des formes, comme la logique, mais sont plus primitives que les déterminations logiques mêmes.

L'objet de cet article est de discuter cinq notions qui peuvent être présentées comme candidates à cette fonction d'universels protologiques pour les langues naturelles : la pluralité des niveaux d' «articulation", l'énoncé complet, la notion d'«ancrage» de l'énoncé à l'énonciarion, le nom propre, la corrélation rhème-thème.

De tels universaux ne sont pas présentés comme des classes de symboles, mais des types de fonctions diversement réalisées dans des structures grammaticales. Les mettre en évidence - ou en découvrir d'autres - devrait être l'une des tâches de la philosophie du langage dans sa relation à la science linguistique.

AHSTRACT. Is it possible to state non-empirical requisites for an object, fact or artefact, to obtain symbolical functioning? If so, their status could be named proto-logical, in so far as they bear upon forms, like logic, but ought to be more primitive than logical determinations.

The purpose of this paper is to discuss five notions which may be considered candidates for the functional role of proto-logical universals of natural languages: plurality of the levels of "articulations ", the concept of full-fledged sentence, "anchorage " of enunciated sentences to enunciation, proper names, the correlation rheme-theme.

1. Cet article est la rédaction d'un exposé présenté aux membres du groupe d'études «Le Mercure philosophique », le 12 novembre 1988 à Sarrebrück. Je remercie mes collègues de ce groupe des observations dont ils ont bien voulu me faire profiter. 
Such universals are not meant to correspond to classes of symbols, but to typical functions, diversely embodied in grammatical structures. To trace them - or others - should be one of the tasks of philosophy of language, in its relationship with linguistic investigations.

\section{LA QUESTION DES CONDITIONS DE LA PENSÉE SYMBOLIQUE}

Peut-on formuler des conditions pour que la réaction d'un organisme vivant soit reconnue comme produit d'une fonction de symbolisation? Plus spécifiquement, peut-on formuler des conditions de la reconnaissance d'une production symbolique comme appartenant à une langue, au sens ordinaire donné à ce mot? Nous essaierons de répondre à la seconde question, en remarquant que les conditions qui sont demandées par la première doivent être des recouvrements partiels de l'ensemble des conditions qui satisferaient à la seconde; autrement dit que le symbolisme linguistique est la manifestation la plus complète, et la plus différenciée, d'une fonction symbolique en général.

Pourquoi conditions «proto-logiques»? Il s'agit de conditions concernant des formes, comme la logique. Mais la structuration logique joue son rôle à un niveau déjà élaboré de la pensée symbolique, comme détermination de formes d'objets en général, et détermination de formes d'inférence. Elle suppose donc déjà satisfaites des conditions premières.

Ce sont en effet des conditions plus primitives qui rendent possible cette structuration. Par exemple : les formes de prédication, plus «profondes», quoique pourtant plus spécifiques, que les formes d'inférence qui, elles, sont de nature logique. Un système symbolique au sens le plus complet, comme la langue ordinaire, ne se réduit pas à un système formel, essentiellement gouverné par des conditions logiques, bien qu'il puisse être décrit comme tel à un certain niveau. De ce point de vue, les systèmes formels apparaissent à la fois comme produits plus élaborés, et comme organisations incomplètes, ne remplissant pas certaines des conditions protologiques constitutives d'une langue ordinaire. Ce sont ces dernières qui nous retiendront, en tant qu'elles rendraient compte de la nature des systèmes symboliques les plus complexes, possédant d'une certaine manière les ressources de tous les autres. 
Pourquoi pas «proto-grammaticales" ? C'est qu'une grammaire décrit l'organisation d'un système symbolique en considérant à un certain niveau des aspects de la substance de l'expression, et de la forme - sinon de la substance - du contenu, dans le langage de Hjelmslev. Une grammaire n'est jamais radicalement formelle. En ce sens, je dirai qu'il ne peut y avoir de " grammaire universelle », bien que la grammaticalité puisse être formulée à des degrés différents d'abstraction, par exemple pour des familles de langues. Les règles chomskyennes concernent des phénomènes attachés à des réalisations substantielles propres à certaines langues, qu'elles traduisent en termes abstraits en occultant leur spécificité. Fausse généralité qui neutralise à un niveau inadéquat le concret des langues.

Les conditions qui nous intéressent sont ainsi, nécessairement et pour ainsi dire a fortiori, proto-grammaticales. Nous continuerons donc de les nommer «proto-logiques », et nous demanderons quel est leur rapport aux grammaires. Les conditions proto-logiques se réalisent sous des formes variées dans les grammaires. Elles seront mises au jour par réflexion, sans être cependant tirées par simple recensement des formes grammaticales et par induction; mais elles seront mises à l'épreuve dans les langues les plus diverses. Il s'agit alors autant de philosophie du langage que de linguistique descriptive; collaboration nécessaire mais difficile, dans la mesure où la linguistique, sous aucune forme, ne saurait se confondre avec une réflexion de philosophe, et où la philosophie, en revanche, ne peut se substituer à la connaissance positive.

Ces conditions proto-logiques renvoient-elles à des conditions biologiques empiriquement décelables? Toute prise de position philosophique de type matérialiste implique la conjecture d'une réponse, en un certain sens, affirmative. Mais l'état actuel de la physiologie cérébrale et de la psychologie du langage ne permettent pas de développer la réponse. L'essai de Thom ${ }^{2}$, par exemple, est décevant ; les propositions de Changeux dans l'Homme neuronal ${ }^{3}$ ( «objets mentaux » comme « entrée en activité corrélée et transitoire d'une assemblée de neurones » p. 174) ne résolvent pas la question

2. R. THом, Modèles matbématiques de la morphogénèse, Paris, Christian Bourgeois, 1980 , ch. X à XV.

3. J.-P. Changeux, L'Homme neuronal, Paris, Fayard, 1983, ch. V. 
de la représentation, du «renvoi. » Quel que soit le progrès des neurosciences, il me semble que leurs réponses laisseront toujours, en deçà, la question philosophique de la possibilité a priori du symbolisme.

Le problème général alors posé est celui d'une délimitation des frontières du comportement symbolique (par exemple, par opposition à un comportement réflexe). Nous laisserons aux biologistes et psychologues du futur la tâche de décrire les structures anatomiques et fonctionnelles qui rendent matériellement possible la symbolisation, ainsi que de retracer l'évolution qui les aura créées. Mais nous prenons les conditions proto-logiques de symbolisation comme transcendantales, constitutives d'une possibilité de penser la fonction, en même temps que signifiant une possibilité de la fonction même.

\section{CINQ UNIVERSAUX PROTO-LOGIQUES}

Je ferai trois remarques préalables:

1) Les universaux que nous allons examiner seront des fonctions plutôt que des classes de symboles ; la réalisation des fonctions par des classes de symboles relèverait des grammaires spécifiques.

2) Leur choix ne doit pas être pris pour une présomption d'énumération exhaustive. Il s'agira d'une liste ouverte: non pas un «système de la sémiotique pure», mais quelques-unes des conditions sine qua non de fonctionnement d'une langue ordinaire, dont certaines ne sont pas remplies par d'autres systèmes symboliques. Il est peut-être impossible de dessiner une ligne de démarcation précise entre les diverses formes de systèmes symboliques qui se déploient, du plus abstrait au plus riche, entre systèmes formels et langues ordinaires (car ces formes sont des concepts philosophiques). C'est pourquoi il serait vain de viser à constituer ces conditions en un système clos. Cette absence de clôture pourrait être objectée au résultat d'une visée qui se veut transcendantale; mais nous n'entendons pas ici par transcendantal une détermination rigide et complète, mais plutôt un ensemble de conditions qui constituent le point de départ d'une pensée minimale de la symbolisation linguistique. 
3) On distinguera fonction de symbolisation et fonction de communication. Toutes deux sont présentes dans les langues ordinaires (qui sont des systèmes de communication symbolique), mais la seconde peut être absente ou secondaire dans d'autres systèmes symboliques (formels par exemple). La première peut également être absente ou secondaire dans des systèmes de communication (la communication animale, certaines formes de communication affective). Nos universaux ne sont donc pas des réquisits de la communication en général ; mais ils sont constitutifs de la communication symbolique. J'en propose cinq, sous les réserves qui viennent d'être indiquées.

\section{La pluralité d'articulation}

1) On empruntera à $A$. Martinet le mot d'articulation. La double articulation des langues ordinaires est évidente: elles comportent un niveau de fractionnement ultime des signes, un niveau d'organisation élémentaire. Les renvois des signes élémentaires ne sont pas des "objets» extérieurs au système. Leur fonction est informationnelle stricto sensu. Ces signes servent à former les signes de niveau supérieur qui renvoient à de tels objets.

Cette articulation, qui suppose donc le caractère discret du signe linguistique, rend possible la création réglée mais non bornée des signes du niveau supérieur. À l'un de ces niveaux, la notion grammaticale de «mot » est fondamentale, dans la mesure justement où elle correspond aux réalisations diverses du signe de niveau supérieur dans les langues: mais cette multiplicité des niveaux d'articulation empêche qu'on puisse décider en général lequel de ces niveaux est organisé en " mots », et d'en donner une définition universelle plus précise sur le plan grammatical.

L'important n'est pas qu'il y ait deux articulations ou davantage, mais c'est la distinction d'une articulation ultime et d'autres articulations : il y a du reste, dans une langue, plus de deux niveaux en général. Cette liberté du nombre des niveaux rend ainsi compte de la mobilité des distinctions entre syntaxe et morphologie et de l'incertitude des classifications en langues agglutinantes/isolantes, analytiques/synthétiques. 
2) La dissociation des articulations peut être codifiée ou non codifiée. L'articulation au niveau du discours est peu ou pas codifiée, sauf dans les usages rituels de la langue (le «rituel» démonstratif euclidien par exemple). Elle n'en existe pas moins, plus ou moins réglée selon les langues et selon les états de langue, ce qui justifie qu'on puisse envisager - quoiqu'avec prudence une linguistique du discours. En revanche, les emplois des niveaux non codifiés de l'articulation, ou des parties libres des niveaux codifiés, est ce qui rend possibles les effets de style.

3) Ce réquisit des systèmes de communication symbolique est-il toujours satisfait dans d'autres systèmes?

Le symbolisme logico-mathématique ne comporte pas, en général, de double articulation. Chaque symbole renvoie à un objet ou à une opération mathématiques. Par exemple, le $d \mathrm{de} \mathrm{dx}$ ne joue pas le rôle d'un phonème dans un monème de la langue : il a un «sens » (opératoire) par lui-même. Il convient, de ce point de vue, de ne pas confondre l'appartenance à un niveau premier d'articulation, et le fait d'être un signe syncatégorématique, car il est bien clair que nombre de signes mathématiques appartiennent à cette dernière classe, sans pour autant être des signes de première articulation. On ne confondra pas davantage avec une première articulation les signes ou marques de «ponctuation » mathématique, ou l'usage d'un ordre numérique ou alphabétique dans les indices, par exemple, car s'ils jouent bien un rôle intrasyntaxique, la syntaxe elle-même appartient ici au sens mathématique.

Que dire des codes: le code morse par exemple? En tant que système symbolique propre, il comporte un seul niveau de signes, celui des points et des traits. L'organisation d'une seconde articulation en mots appartient non au code en tant que tel mais à la langue véhiculée, que le code décalque.

Pour les écritures alphabétiques, en tant que codes, même situation. La situation des écritures idéographiques est plus complexe, et labile: les caractères chinois comportent partiellement une seconde articulation (les clefs seraient éléments de l'articulation primaire); ils fonctionnent du reste presque comme une vraie langue en ce qui concerne le chinois classique, du moins pour le 
lecteur moderne, alors que dans leur usage actuel ils servent à coder la langue parlée moderne. Une question cependant : peut-on pousser (artificiellement) plus loin la dissociation d'une articulation primaire dans les symbolismes idéographiques? Par exemple décomposer les caractères pour en normaliser l'écriture mécanique? Certainement, puisque le processus est déjà en germe dans le recensement calligraphique des espèces de traits et de points.

\section{La fonction d'énoncé complet}

Certains groupes de symboles sont désignés en français comme «phrases » ou «propositions ». Dans un système formel, il est possible d'en donner une définition explicite, soit sémantique, soit syntaxique. Du premier point de vue on dira qu'un énoncé complet est toujours ou vrai ou faux ${ }^{4}$ (Aristote); du second, on donnera récursivement le moyen de construire des énoncés complets à partir des symboles (la forme canonique de la proposition d'A ristote, celle des calculs logiques classiques), en nombre fini, du vocabulaire primitif. Mais cette possibilité masque la véritable nature de l'énoncé complet, car les deux procédés ne sauraient réussir qu'occasionnellement dans les langues ordinaires. La fonction d'énoncé complet est en effet antérieure à la dissociation grammaticale du sémantique, du syntaxique et du pragmatique. Si l'on projette cette distinction sur la notion d'énoncé complet dans un symbolisme déterminé, on pourra sans doute discerner a posteriori des traits qui le caractérisent de l'un ou de plusieurs de ces points de vue, mais qui varieront en général selon le système considéré. Nous disons donc que cette fonction est un indéfinissable protologique, qui se réalise explicitement dans les différents systèmes, sous les espèces d'une catégorie grammaticale.

Je ferai cependant deux remarques, essentiellement négatives, concernant la nature de cette fonction.

4. Nous ne parlons ici que de l'énoncé complet d'un système formel, et n'envisageons pas, par conséquent, les formes interrogatives, impératives, etc. Il sera dit plus loin que l'énoncé complet des langues naturelles appelle des spécifications, modales en un sens large, et de nature variée. 
$1^{\circ}$ En tant que proto-logique, l'énoncé complet ne saurait être divisé comme tel selon des formes de prédication, spécification qui fait intervenir des prises de position concernant la forme du contenu et permet l'élaboration de diverses métaphysiques. Ces formes de prédication spécifiques ne peuvent appartenir quau régime d'organisation grammatical. Mais c'est la nécessité de spécifier des formes de prédication qui constitue, sur ce point, l'aspect principal de cette condition proto-logique. Nous verrons plus loin qu'un autre universel proto-logique, l'opposition thèmerhème, peut intervenir dans la spécification grammaticale de l'énoncé complet. Bien entendu, d'autres registres de spécification se présentent, en particulier dans les langues naturelles, ou une apostrophe, un ordre, une exclamation constituent assurément des réalisations d'énoncés complets.

$2^{\circ}$ Que dire des modalités, par exemple, qui sont certainement, au moins dans les langues ordinaires, des réalisations spécifiques d'énoncés complets? Elles spécifient, au niveau de la langue, l'aspect forme d'assertion, et la fonction énoncé complet exige cette spécification. On notera qu'une telle spécification peut fort bien consister en une neutralisation, comme c'est le cas dans certains systèmes formels. Il me semble, du reste, que cette neutralisation est l'un des traits caractéristiques du système qui mérite le nom de logique stricto sensu (où l'on constate également la neutralisation de la pluralité des formes de prédication); de sorte que les «logiques» modales, au contraire, sont des théories d'un monde, il est vrai très abstrait, mais d'un monde, avec des objets déjà qualifiés ; elles relèvent d'une formalisation, certes très réductrice, du langage ordinaire bien plutôt que de la logique.

Il appartient aux linguistes de décrire pour une langue ou un groupe de langues, par des traits qui peuvent relever des trois distinctions grammaticales - syntaxe, sémantique, pragmatique les spécifications très diverses de la fonction énoncé complet. Mais c'est malheureusement trop souvent le cas aujourd'hui, parce qu'ils ne s'intéressent qu'à des langues assez voisines ou même à une seule langue, ou encore parce qu'ils confondent inopportunément philosophie du langage et linguistique, que cette diversité extrêmement intéressante échappe à certains linguistes. 


\section{La fonction d'« ancrage»}

Dans une langue ordinaire, je dis qu'il faut pouvoir exprimer autrement qu'en la décrivant de l'extérieur la situation du locuteur. C'est à quoi répondent par exemple notre pronom $j e$, mais aussi les mots démonstratifs qui désignent les objets du monde à partir de la position occupée par ce je. De savoir comment se réalise dans une langue donnée cette fonction que je nomme d' «ancrage » (à la position du locuteur dans son monde), comment elle fait intervenir un jeu plus ou moins compliqué de référence à d'autres actants (toujours situés par rapport à je), c'est le travail du linguiste. Notre hypothèse est que, bien entendu, ces symboles, «indicateurs de subjectivité » ont effectivement dans une langue un statut distingué, que leur grammaire a au moins quelque trait singulier, correspondant aux diverses réalisations d'une fonction proto-logique commune. On peut d'autre part conjecturer qu'ils apparaissent dans toute langue naturelle, alors que l'on constate leur absence dans d'autres systèmes symboliques.

Mais de tels symboles ne peuvent guère se distinguer par des propriétés grammaticales de type sémantique ou syntaxique. Certes, le renvoi du pronom je, par exemple, est mobile, et autrement que ne l'est celui du mot "étoile». Mais ce caractère proprement sémantique dérive directement du statut pragmatique du mot $j e$ : c'est parce que le renvoi du je est lié à l'énonciation de l'énoncé où il entre qu'il y a mobilité de son sens. Cependant, certaines langues possèdent des systèmes de marques syntactico-sémantiques de l'ancrage, signalant explicitement la position et la dignité relative du locuteur et des actants. Ce ne sont là assurément que des avatars grammaticaux de la fonction fondamentale.

\section{La fonction nom-propre}

La fonction nominale, réalisée en nos langues par les noms «communs » est évidemment essentielle à tout symbolisme. On peut dire que, d'une certaine manière, tout symbole fonctionne d'abord comme "nom », puisqu'il renvoie à quelque chose, fût-ce à un autre symbole. C'est en ce sens que je ne recense pas le nom parmi les universaux. La fonction nom-propre me paraît, au contraire, en être suffisamment distincte pour qu'il faille la proposer comme une condition proto-logique séparée. 
Je me permets de renvoyer sur ce point à l'article: «À quoi servent les noms propres?» (in Langages, $\mathrm{n}^{\prime \prime} 66$, juin 82, p. 21-36). Je me bornerai ici à relever d'abord un trait, certes important du nom propre, mais qui n'est qu'illusoirement déterminant de la fonction: à savoir l'unicité de désignation. Car si c'était une détermination suffisante, le nom propre pourrait être remplacé par une description définie.

Mais ne peut-il pas l'être par un indicateur de subjectivité (par un marqueur d'ostension)? Dans une certaine mesure, oui ; mais le nom propre suppose la stabilité du désigné (désignateur «rigide»), contrairement à l'indicateur de subjectivité.

Le trait déterminant que nous retenions finalement dans l'article cité est la possibilité de servir à une interpellation virtuelle, caractère dont la réalisation grammaticale - pragmatique serait essentielle à la fonction nom-propre. C'est ce qui rend compte entre autres du fait que n'importe quel symbole, n'importe quel mot d'une langue peut être détourné de sa fonction originaire et utilisé comme nom propre; ce sont les législations et les coutumes tribales qui tendent à fixer cette fonction dans une classe fermée (la liste des noms de baptême).

Le nom propre a-t-il un sens? Dans le vocabulaire de Peirce je le caractérise comme index-symbol, c'est-à-dire comme une règle de désignation ancrée dans une énonciation. Il faudrait donc dire non que le nom propre a nécessairement comme tel un sens, mais qu'il comporte une présupposition de sens, et qu'il importe un halo de connotations, qui en explique les vertus poétiques latentes. D'autre part, des règles et coutumes sociales, plus ou moins contraignantes et minutieuses, peuvent en régler tellement l'usage qu'il acquiert ainsi des sens classificatoires dans le groupe. Mais la fonction de porteur de sens est originairement vicariante.

On observera enfin que la fonction nom-propre est totalement absente des systèmes formels, qui comportent tout au plus des descriptions définies abrégées en symboles donnant l'illusion d'être des noms propres: tels $\pi$, e, la fonction $\zeta \ldots$ Mais on n'a même pas de désignation abrégée, de faux nom propre, pour d'autres, telle "la constante d'Euler». Cette fonction nom-propre joue au contraire un rôle essentiel dans la communication et dans la création esthétique, où l'interpellation virtuelle est importante. 


\section{La fonction d'opposition rhème-thème}

C'est l'opposition entre ce que l'on dit (le «rhème») et ce dont on le dit (le " thème »). Quel est son rapport à la prédication, que nous avons proposé de considérer, dans ses formes spécifiques, comme concernant la grammaire de chaque langue? Nous le caractériserions de la manière suivante:

La corrélation «sujet»/ «prédicat» est interne à l'énoncé complet (et particulièrement à l'énoncé complet déclaratif) qui, dans chaque langue, en spécifie nécessairement la nature selon les moyens propres à une grammaire.

La corrélation « rhème »/ «thème » se situe au contraire dans un cadre qui peut déborder l'énoncé complet. En revanche, il arrive que certaines langues peuvent, il est vrai, déterminer des énoncés complets sans lien de prédication, par une simple opposition, alors interne, du type rhème-thème, ou encore superposent une opposition thème/rhème à une corrélation prédicative ; mais cette opposition opère en général sur des unités de discours plus larges.

Au niveau des grammaires, cette fonction proto-logique est marquée de façon plus ou moins explicite et réglée. En français, elle est marquée, quand elle l'est en langue, par des accents d'intensité prosodique, ou par des tournures syntaxiques, comme les formules commençant par : «c'est... ». En japonais, par contre, il existe un système explicite explicitant cette opposition au moyen des particules $g a$ et $w a$, qui servent à accentuer le thème ou à l'affaiblir. C'est ainsi que ga concentre l'attention sur les mots qui précèdent (le rhème), wa relâchant l'attention vers d'autres parties de la phrase. On le voit sur l'exemple suivant emprunté à S. Martin, Essential Japanese, (C.E. Tutle, Tokyo, 3 rd. ed., 1972, pp. 44-45) :

"éiga ga suki desu» : j'aime le cinéma, c'est le cinéma que j'aime

«éiga wa suki desu»: j’aime le cinéma, le cinéma, je l'aime.

Cette corrélation est absente des systèmes formels et des grammaires des «langages» de la science. Mais elle est réalisée quand il convient par des périphrases (en langue naturelle), ou par des dispositions graphiques ad boc.

On peut se demander s'il est bien légitime de la placer sur le même plan que les universaux précédemment proposés. S'il m'a 
semblé qu'il fallait donner à cette question une réponse affirmative, c'est que l'opposition du thème au rhème m'a paru plus profonde que l'opposition prédicative sujet-prédicat, de nature grammaticale, apparemment si naturelle à nos langues, mais à laquelle l'autre corrélation peut parfaitement se substituer. Resterait à découvrir des systèmes linguistiques où cette substitution est usuelle sinon totale; mais il est d'abord essentiel qu'elle puisse être pensée.

La satisfaction de tels réquisits « proto-logiques » se manifeste par des propriétés, des règles et des contraintes spécifiques, apparentes au niveau des systèmes de signes, au niveau des "grammaires».

Ces réalisations grammaticales sont essentiellement multiples, et il n'y a pas lieu d'espérer établir une grammaire universelle.

Ce qui peut en tenir lieu, c'est justement le recensement et l'analyse de ces réquisits, qui est une tâche philosophique. Mais la relation de la philosophie du symbolisme aux théories empiriques, comme la linguistique ou certaines parties de l'esthétique, consisterait alors en ceci que toute proposition d'une condition proto-logique de symbolisation devrait être appuyée par l'exhibition de plusieurs réalisations grammaticales significativement distinctes. Corrélativement, les règles établies par la grammaire descriptive d'une langue devraient pouvoir être rattachées à un réquisit protologique.

Collège de France 